\title{
The elasto-plastic Point Method to estimate fatigue lifetime of notched metallic materials under variable amplitude multiaxial fatigue loading
}

\author{
Namiq Zuhair Faruq, Luca Susmel ${ }^{\square}$ \\ Department of Civil and Structural Engineering, The University of Sheffield, \\ Mapping Street, Sheffield S1 3JD, UK
}

\begin{abstract}
The present paper deals with the formulation and implementation of a novel fatigue lifetime estimation technique suitable for designing notched components against multiaxial fatigue. This fatigue assessment procedure was devised by combining the Modified Manson-Coffin Curve Method and the Shear Strain-Maximum Variance Method with the elastoplastic Point Method. The accuracy of the approach being proposed was checked against a large number of experimental results that were generated by testing notched cylindrical samples of medium-carbon steel En8. These tests were run under proportional/non-proportional constant/variable amplitude biaxial loading, with the effect of non-zero mean stresses and different frequencies between the axial and torsional stress/strain components being also investigated. The results from this validation exercise demonstrate that the novel multiaxial fatigue assessment methodology being proposed is highly accurate, with its systematic usage resulting in predictions falling within an error factor of 2 . This remarkable level of accuracy is very promising especially in light of the fact that this approach can be applied by directly post-processing the results from elastoplastic Finite Element (FE) models solved using commercial codes.
\end{abstract}

\section{Introduction}

Engineering components and structures are characterised by complex geometries that lead to localised stress/strain concentration phenomena. Further, during in-service operations, they are subjected to load histories that are not only multiaxial, but also vary over time randomly. This is the reason why systematic research work has been carried out worldwide since the beginning of the $1900 \mathrm{~s}$ to formulate and validate reliable procedures suitable for addressing such complex design problem.

As to the different fatigue assessment philosophies that are available to date, examination of the state of the art shows that, in general, stress-based approaches are used to estimate damage in the medium/high-cycle fatigue regime - i.e., when cyclic plasticity can be

${ }^{\square}$ Corresponding author: 1.susmel@ sheffield.ac.uk 
neglected with little loss of accuracy. In contrast, strain-based approaches are usually preferred whenever metallic materials undergo large scale cyclic plastic deformations [1-3].

If attention is focussed on the problem of designing against multiaxial fatigue notched metallic materials failing in the low/medium-cycle fatigue regime, root stresses and root strains post-processed according to the critical plane concept are seen to return accurate estimates [4-6], with this holding true provided that the notches being designed are relatively blunt [5]. In contrast, when the geometrical features under investigation are sharp, strainbased approaches return estimates that are somehow too conservative, with the level of conservatism increasing as the sharpness of the considered notch increases $[7,8]$.

In this scenario, to extend effectively the use of the strain-based approach to those situations involving stress/strain gradients resulting not only from blunt, but also from sharp geometrical features, this paper aims at formulating and validating a novel fatigue lifetime estimation technique based on the following three key ingredients: (i) the Modified MansonCoffin Curve Method (MMCCM) [3, 5, 9], (ii) the Shear Strain-Maximum Variance Method $(\gamma$-MVM) [3, 10, 11], and (iii) the elasto-plastic Point Method (PM) [7, 8].

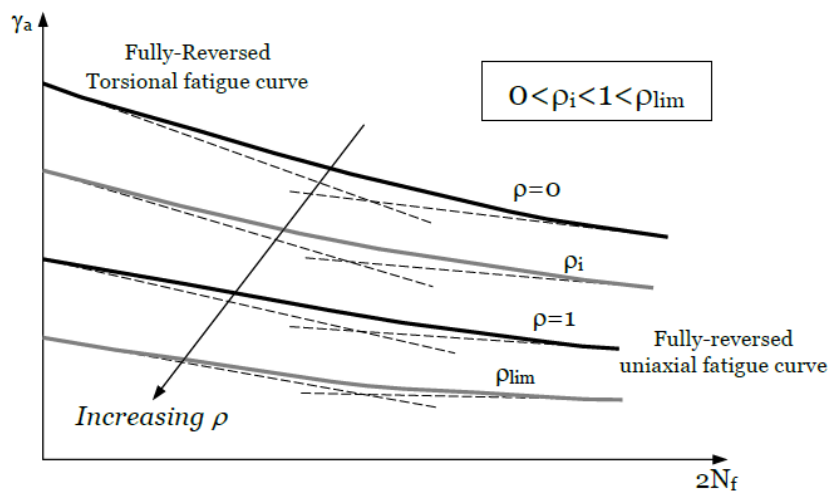

Fig. 1. The modified Manson-Coffin diagram.

\section{Fundamentals of the MMCCM}

The MMCCM postulates that fatigue lifetime can be predicted accurately by using nonconventional bi-parametrical Manson-Coffin curves [9]. The formulation of this criterion takes as a starting point the assumption that fatigue damage can be assessed in log-log diagrams that plot the shear strain amplitude, $\gamma_{\mathrm{a}}$, relative to that plane experiencing the maximum shear strain amplitude (i.e., the so-called critical plane) against the number of reversals to failure, $2 \mathrm{~N}_{\mathrm{f}}$ (Fig. 1). According to the schematic chart reported in Fig. 1, the equation describing any Modified Manson-Coffin curve can directly be expressed as $[3,9]$ :

$$
\gamma_{a}=\frac{\tau_{f}^{\prime}(\rho)}{G} \cdot\left(2 \cdot N_{f}\right)^{b(\rho)}+\gamma_{f}^{\prime}(\rho) \cdot\left(2 \cdot N_{f}\right)^{c(\rho)}
$$

where critical plane stress ratio $\rho$ is defined as follows:

$$
\rho=\frac{\sigma_{n, m}+\sigma_{n, a}}{\tau_{a}}=\frac{\sigma_{n, \max }}{\tau_{a}}
$$

In Eq. (2) $\tau_{\mathrm{a}}$ is the shear stress amplitude relative to the plane of maximum shear strain amplitude, whilst $\sigma_{\mathrm{n}, \mathrm{m}}, \sigma_{\mathrm{n}, \mathrm{a}}$ and $\sigma_{\mathrm{n}, \max }$ are the mean value, the amplitude and the maximum value of the stress perpendicular to the critical plane, respectively. It is worth recalling here that, thanks to the way it is defined, ratio $\rho$ is sensitive not only to the degree of multiaxiality 
and non-proportionality of the applied loading path, but also to the presence of superimposed static stresses $[3,9,11]$. Turning back to Eq. (1), functions $\tau_{f}^{\prime}(\rho), \gamma_{f}^{\prime}(\rho), b(\rho)$ and $c(\rho)$ can be estimated using the following relationships [9]:

$$
\begin{gathered}
\frac{{\tau^{\prime}}_{f}(\rho)}{G}=\rho \cdot\left(1+v_{e}\right) \frac{\sigma_{f}^{\prime}}{E}+(1-\rho) \frac{\tau_{f}^{\prime}}{G} ; \gamma_{f}^{\prime}(\rho)=\rho \cdot\left(1+v_{p}\right) \varepsilon_{f}^{\prime}+(1-\rho) \gamma_{f}^{\prime} \\
b(\rho)=\frac{b \cdot b_{0}}{\left(b_{0}-b\right) \rho+b} ; c(\rho)=\frac{c^{\cdot} c_{0}}{\left(c_{0}-c\right) \rho+c}
\end{gathered}
$$

By following a fairly articulated reasoning, calibration functions (3) and (4) were derived by directly using the conventional fully-reversed uniaxial and torsional Manson-Coffin fatigue curves re-written in terms of maximum shear strain amplitude, i.e. [3,9]:

$$
\begin{gathered}
\left.\gamma_{a}=\left(1+v_{e}\right) \frac{\sigma_{f}^{\prime}}{E}\left(2 \cdot N_{f}\right)^{b}+\left(1+v_{e}\right) \varepsilon_{f}^{\prime}\left(2 \cdot N_{f}\right)^{c} \text { (Uniaxial case, } \rho=1\right) \\
\left.\gamma_{a}=\frac{\tau_{f}^{\prime}}{G}\left(2 \cdot N_{f}\right)^{b_{0}}+\gamma_{f}^{\prime}\left(2 \cdot N_{f}\right)^{c_{0}} \text { (Torsional case, } \rho=0\right)
\end{gathered}
$$

where $v_{\mathrm{e}}$ and $v_{\mathrm{p}}$ are Poisson's ratio for elastic and plastic strain, respectively.

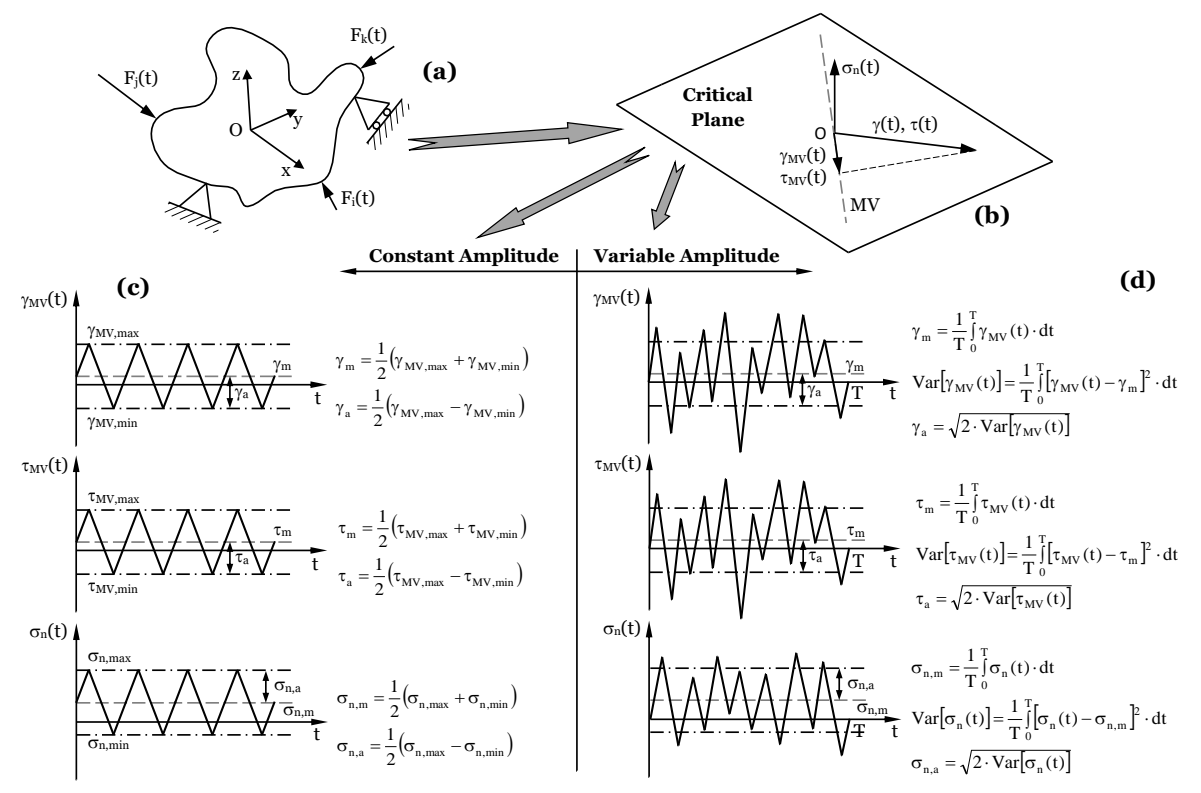

Fig. 2. Critical plane Stress/strain components determined according to the $\gamma$-MVM.

To estimate the orientation of those material planes experiencing the maximum shear strain amplitude, the MMCCM is recommended to be used along with the $\gamma$-MVM [10, 11$]$. The $\gamma$-MVM defines the orientation of the critical plane via that direction, MV, that is associated with the maximum variance of the resolved shear strain, $\gamma_{M V}(t)$ - see Figs $2 a$ and $2 \mathrm{~b}$. As far as variable amplitude (VA) load histories are concerned, the key advantage of the $\gamma$-MVM is that the shear stresses and shear strains relative to the critical plane are managed and quantified via resolved quantities $\tau_{M V}(t)$ and $\gamma_{M V}(t)$, respectively (Fig. $\left.2 d\right)$. Since, by definition, these two quantities are monodimensional stress/strain signals, fatigue cycles under multiaxial fatigue loading can be counted rigorously and effectively by simply using the well-known Rain-Flow counting method that was originally formulated to specifically address the uniaxial fatigue problem. 


\section{Multiaxial notch fatigue assessment: the proposed approach}

The multiaxial fatigue life estimation methodology proposed in the present paper is based on the use of the MMCCM, the $\gamma$-MVM, and the elasto-plastic PM. The key features of this design approach will be explained in the following two sections by considering constant amplitude (CA) and variable amplitude (VA) load histories, respectively. For the sake of clarity, the multiaxial fatigue assessment approach being proposed will be formulated by assuming that the critical distance, L, needed to use the PM is known a priori, with the procedure suitable for determining $\mathrm{L}$ being explained in Section 3.3.

\subsection{Fatigue assessment under CA multiaxial fatigue loading}

Figure 3 summarises the design methodology that is proposed to estimate CA fatigue lifetime of notched components by applying the MMCCM along with the elasto-plastic PM.

Consider the notched structural detail sketched in Figure $3 \mathrm{a}$ and assume that, during inservice operations, it is subjected to a complex system of CA time-variable external forces and moments.

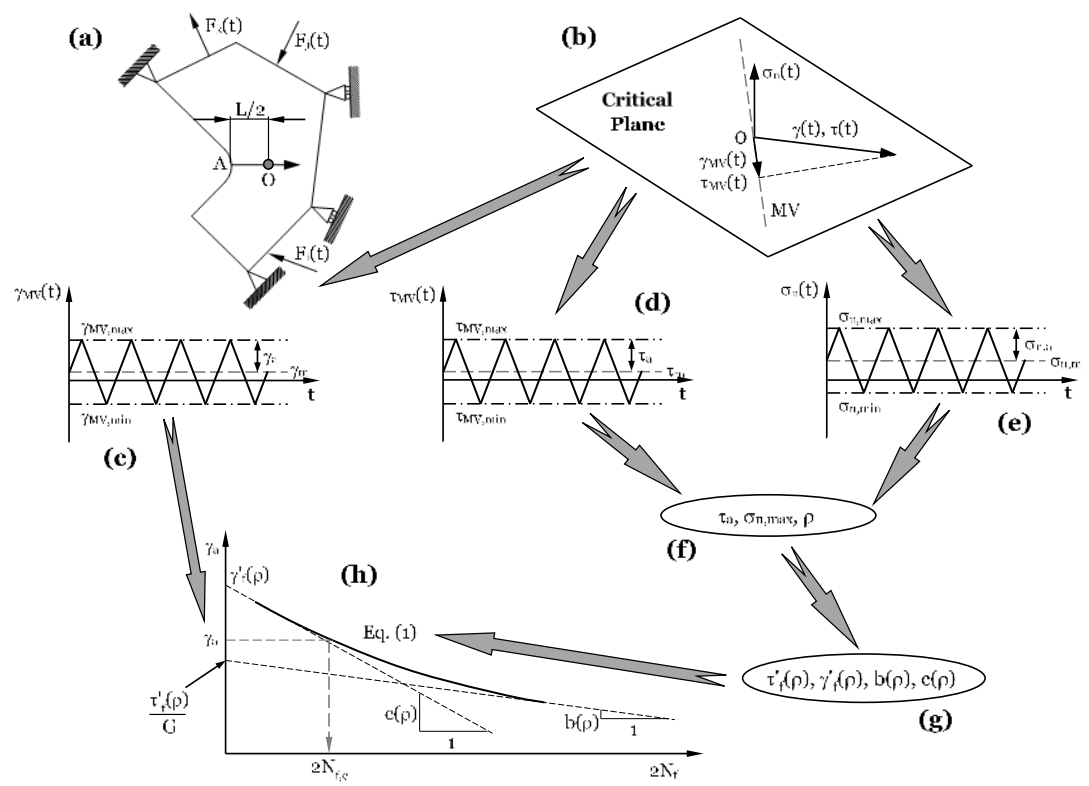

Fig. 3. Procedure to design notched components against CA multiaxial fatigue loading.

To use the MMCCM in conjunction with the PM, the first task is the determination of the elasto-plastic cyclic stress/strain fields along the so-called focus path. The focus path is defined as a straight line that emanates from the assumed crack initiation point (i.e., point A in Figure 3a) and is normal to the component surface at the hot spot itself [3]. According to the PM, the relevant stress/strain quantities needed to estimate fatigue lifetime have to be determined by post-processing the cyclic stress tensor and the cyclic stain tensor determined, along the focus path, at a distance from the assumed crack initiation point equal to $\mathrm{L} / 2$ (i.e., at point $\mathrm{O}$ in Figure 3a). The orientation of the critical plane is determined from the timevariable strain tensor at point $\mathrm{O}$ via the $\gamma$-MVM. As soon as the direction, $\mathrm{MV}$, of maximum variance of the resolved shear strain is known (Fig. 3b), $\gamma_{\mathrm{a}}$ (Fig. $3 \mathrm{c}$ ) and $\tau_{\mathrm{a}}$ (Fig. $3 \mathrm{~d}$ ) can be determined directly according to the standard definitions reported in Fig. 2c. In a similar way, 
by post-processing stress signal $\sigma_{\mathrm{n}}(\mathrm{t})$, the mean value and the amplitude of the stress perpendicular to the critical plane can be calculated unambiguously (Fig. 3e) by using the definitions summarised in Fig. 2c.

The values for $\tau_{\mathrm{a}}, \sigma_{\mathrm{n}, \mathrm{m}}$, and $\sigma_{\mathrm{n}, \mathrm{a}}$ being determined via the $\gamma$-MVM are then used to quantify stress ratio $\rho$ (Fig. 3f). By so doing, the corresponding modified Manson-Coffin curve, Eq. (1), can be estimated directly via calibration functions (3) and (4) - see Fig. 3g. Finally, as shown in the Modified Manson-Coffin diagram sketched in Figure 3h, it is straightforward to estimate the number of reversals to failure, $2 \mathrm{~N}_{\mathrm{f}}$, via the modified Manson-Coffin curve being determined according to Eq. (1) and calibrated via relationships (3) and (4).

\subsection{Fatigue assessment under VA multiaxial fatigue loading}

Consider the notched structural detail sketched in Fig. 4a and assume that this component is subjected to a complex system of external VA forces and moments. As per the CA case, also in the presence of VA load histories the first problem to be addressed is the determination of the elasto-plastic cyclic stress/strain fields along the focus path so that the required stress/strain tensors can determined at a distance from the assumed crack initiation point equal to L/2 (Fig. 4a). Subsequently, the strain tensor being estimated is post-processed according to the $\gamma$-MVM in order to determine that direction, MV, which experiences the maximum variance of the resolved shear strain (Fig. 4b).

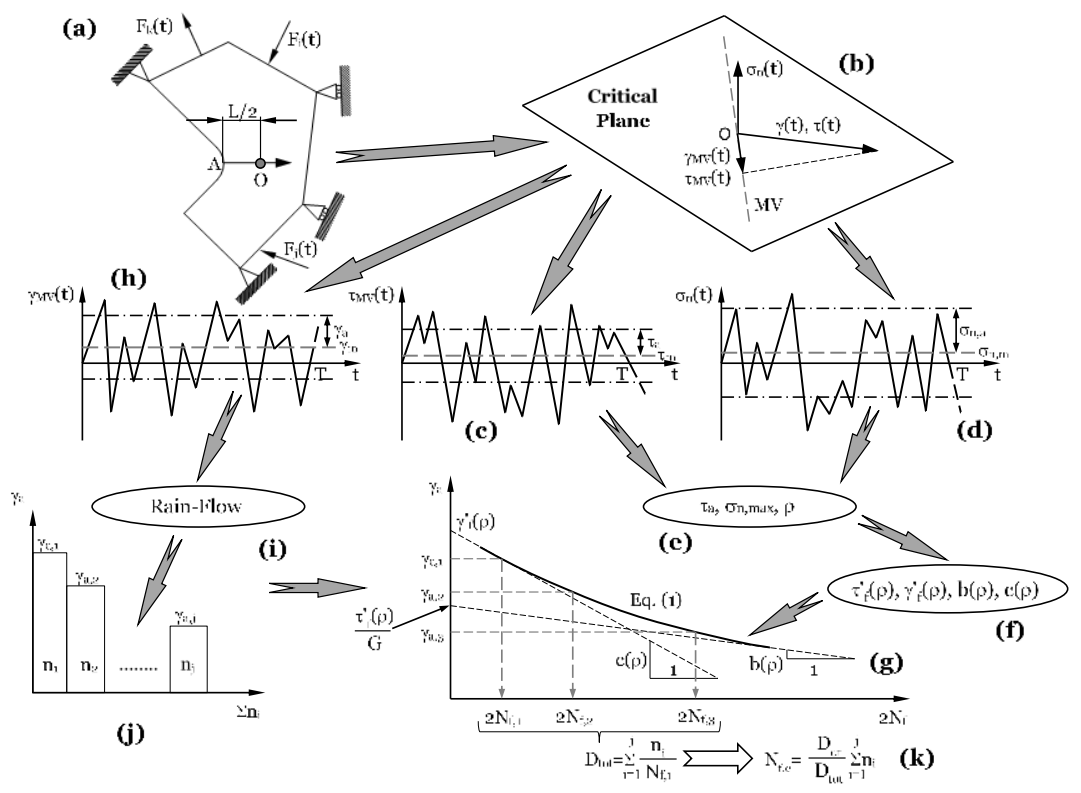

Fig. 4. Procedure to design notched components against VA multiaxial fatigue loading.

As soon as the orientation of the critical plane is known (Fig. 4b), the equivalent amplitude of the shear stress, $\tau_{\mathrm{a}}$, as well as the mean value, $\sigma_{\mathrm{n}, \mathrm{m}}$, and the equivalent amplitude, $\sigma_{\mathrm{n}, \mathrm{a}}$, of the normal stress can directly be determined according to the definitions reported in Fig. $2 \mathrm{~d}-$ see also Figs $4 \mathrm{c}$ and $4 \mathrm{~d}$. By so doing, equivalent stress components $\tau_{\mathrm{a}}$ and $\sigma_{\mathrm{n} \text {,max }}$ [11] are used to determine, via definition (2), the value of the critical plane stress ratio, $\rho$, characterising the VA fatigue load history being assessed (Fig. 4e). Stress ratio $\rho$ together with functions (10) allow then the constants in the MMCCM's governing equation to be estimated directly (Fig. 4f). 
After estimating the modified Manson-Coffin curve to be used to assess fatigue damage (Fig. 4g), the subsequent task is employing the Rain-Flow method to identify and count the cycles from signal $\gamma_{\mathrm{MV}}(\mathrm{t})$, with this allowing the shear strain spectrum associated with the load history under investigation to be determined unambiguously (Figs $4 \mathrm{~h}$ and $4 \mathrm{i}$ ). The obtained shear strain spectrum (Fig. 4j) together with the modified Manson-Coffin curve estimated via Eq. (1) are then used to quantify the extent of damage associated with the shear strain cycles being counted (Fig. 4g), with the number of cycles to failure being estimated as follows (Fig. 4k):

$$
D_{t o t}=\sum_{i=1}^{j} \frac{n_{i}}{N_{f, i}} \Rightarrow N_{f, e}=\frac{D_{c r}}{D_{t o t}} \sum_{i=1}^{j} n_{i}
$$

To conclude, it is worth recalling here that in the above relationships, $\mathrm{D}_{\text {tot }}$ is the total value of the damage sum, whereas $\mathrm{D}_{\mathrm{cr}}$ is the critical value for $\mathrm{D}_{\text {tot }}$ that results in the initiation of a fatigue crack in the notched component being assessed.

\subsection{Determination of the PM critical distance}

Figure 2c summarises the procedure we recommend as being used for the experimental determination of critical distance L. To do so, the uniaxial and torsional fatigue properties of the un-notched material under investigation are supposed to be known from the experiments.

Consider now the notched specimen of Fig. 5 and assume that this sample is subjected to a fully-reversed nominal uniaxial loading. Under the applied CA loading path, this notched specimen is seen to fail at a number of cycles to failure equal to $\mathrm{N}_{\mathrm{f}, \mathrm{c}}$.

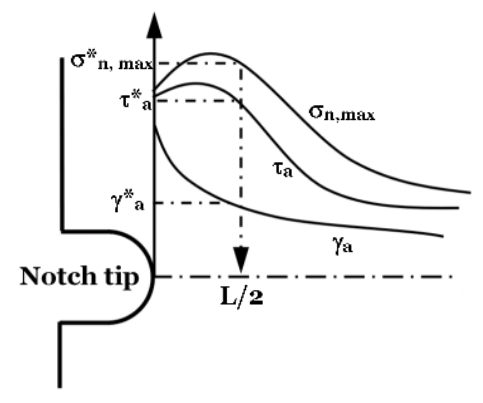

Fig. 5. Determination of the critical distance L according to the Point Method.

The stress/strain-distance curves along the focus path associated with the investigated notched geometry are to be determined by directly post-processing the results from an elastoplastic Finite Element (FE) model. Subsequently, the stress and strain components along the focus path are re-analysed according to the $\gamma$-MVM so that the distribution of $\gamma_{\mathrm{a}}, \tau_{\mathrm{a}}, \sigma_{\mathrm{n}, \max }$, and $\rho$ along the focus path itself can be determined unambiguously (Fig. 2c). Finally, according to the PM $[7,8], \mathrm{L} / 2$ is the distance from the notch tip at which the shear strain amplitude, $\gamma_{\mathrm{a}}{ }^{*}$, is equal to the shear strain amplitude estimated from the MMCCM, Eq. (1), that causes failure in the plain material at $\mathrm{N}_{\mathrm{f}}=\mathrm{N}_{\mathrm{f}, \mathrm{c}}$ (Fig. 5).

\section{Validation by experimental data}

To check the accuracy of the multiaxial notch fatigue design approach proposed in the present paper, an extensive experimental investigation involving both plain and U-notched specimens was carried out at the Structural Integrity Laboratory of the University of 
Sheffield, UK. The specimens being tested were made of En8, i.e., a medium-carbon steel having ultimate tensile stress equal to $701 \mathrm{MPa}$, yield stress to $453 \mathrm{MPa}$, Young's modulus to $210 \mathrm{GPa}$, and shear elastic modulus equal to $80 \mathrm{GPa}$.

The notched cylindrical specimens being tested had net diameter equal to $18 \mathrm{~mm}$, gross diameter to $38 \mathrm{~mm}$ and notch root radius, $\mathrm{r}$, equal to $1.5 \mathrm{~mm}$, to $3 \mathrm{~mm}$, and to $6 \mathrm{~mm}$.

Both plain and notched specimens were tested by using a SCHENCK servo-controlled closed-loop axial-torsion fatigue machine with maximum axial load capacity of $400 \mathrm{kN}$ and maximum torque capacity of $1000 \mathrm{Nm}$. The cylindrical specimens were clamped using two MTS 646.25S hydraulic collet grips. The strain-controlled tests as well as the force/torquecontrolled tests were run under CA/VA sinusoidal loading signals, with the frequency varying in the range $0.5-2 \mathrm{~Hz}$. The experimental results obtained by testing the un-notched specimens were generated by controlling the axial and the shear strains via an Epsilon 3550$025 \mathrm{M}$ bi-axial extensometer with gauge length equal to $25 \mathrm{~mm}$. The number of cycles/blocks to failure was defined both for the plain specimens and the notched samples by $5 \%$ axial/torsional stiffness drop, with the stiffness decrease being estimated under the maximum amplitude of the strain/force applied in the loading path.

By adopting the concave upwards spectrum with sequence length, $\mathrm{S}_{\mathrm{L}}$, of 50 cycles that is shown in Fig. 6a, the VA fatigue behaviour of the plain and notched specimens being tested was investigated by controlling the axial/shear strain and the axial/torsional loading, respectively. In the spectrum of Fig. 6a, given either the axial force, the torque, the axial strain, or the shear strain, $\Omega_{\mathrm{a}, \mathrm{i}}$ denotes the amplitude of the reference strain/loading quantity characterising the $\mathrm{i}$-th cycles, whereas $\Omega_{\mathrm{a} \text {,max }}$ denotes the maximum value in the spectrum of the amplitude of the strain/loading quantity of interest. The tests under VA loading were run by applying the cycles in random order.

The experimental values of the fully-reversed uniaxial cyclic mechanical properties of the carbon steel being tested were as follows: $\mathrm{K}^{\prime}=971.5 \mathrm{MPa}, \mathrm{n}^{\prime}=0.188, \sigma_{\mathrm{f}}{ }^{\prime}=852.3 \mathrm{MPa}$, $\mathrm{b}=-0.105, \varepsilon_{\mathrm{f}}{ }^{\prime}=0.477$, and $\mathrm{c}=-0.554$. The corresponding mechanical properties under fullyreversed torsion were instead as follows: $\mathrm{K}_{0}{ }^{\prime}=442.4 \mathrm{MPa}, \mathrm{n}_{0}{ }^{\prime}=0.107, \tau_{\mathrm{f}}{ }^{\prime}=460.6 \mathrm{MPa}$, $\mathrm{b}_{0}=-0.068, \gamma_{\mathrm{f}}^{\prime}=1.55$, and $\mathrm{c}_{0}=-0.648$. These material constants were used to calibrate the MMCCM - i.e., relationships (3) and (4) - for the specific carbon steel being investigated.

Having calibrated the MMCCM, initially we checked its accuracy in estimating fatigue lifetime in the absence of notches when the required stress/strain quantities relative to the critical plane are determined according to the $\gamma$-MVM. The results from this initial validation exercise are summarised in the experimental, $\mathrm{N}_{\mathrm{f}}$, vs. estimated, $\mathrm{N}_{\mathrm{f}, \mathrm{e}}$, number of cycles to failure diagram reported in Fig. 6b. According to Palmgren and Miner [1, 2], the predictions under VA loading shown in Fig. 6b were obtained by taking the critical value of the damage sum, $D_{\text {cr }}$, invariably equal to unity. This error chart demonstrates that the use of our critical plane approach resulted in estimates falling within an error factor of 2, i.e., within a scatter band as wide as the one containing the fatigue results generated under axial and torsional CA fatigue loading and used to calibrate the MMCCM itself.

In order to post-process the experimental results we generated by testing the notched specimens, FE code ANSYS ${ }^{\circledR}$ was used to estimate the time-variable elasto-plastic stress/strain fields being required to apply the PM. To this end, the notched specimens were modelled using three-dimensional 8-node structural element SOLID185, with the elastoplastic behaviour of the tested steel being simulated by using a multi-linear kinematic rule. After performing conventional convergence analyses, a very refined mesh was used to model the material in the vicinity of the tips of the notches being investigated.

The critical distance value for the tested carbon steel was determined experimentally by applying the PM along with the MMCCM according to the procedure shown in Fig. 5. By so doing, the results generated by testing six sharply notched specimens under uniaxial fatigue loading returned an average value for $\mathrm{L} / 2$ of $0.78 \mathrm{~mm}$. 


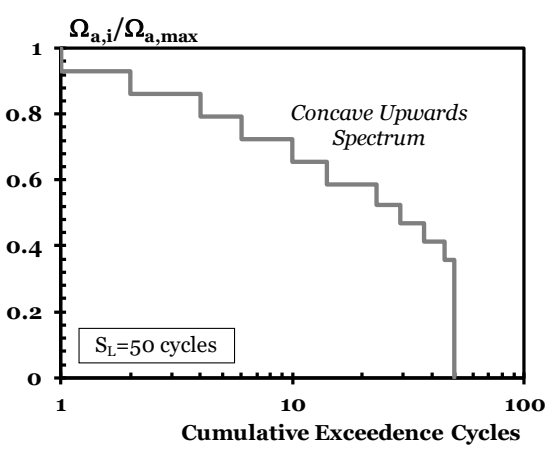

(a)

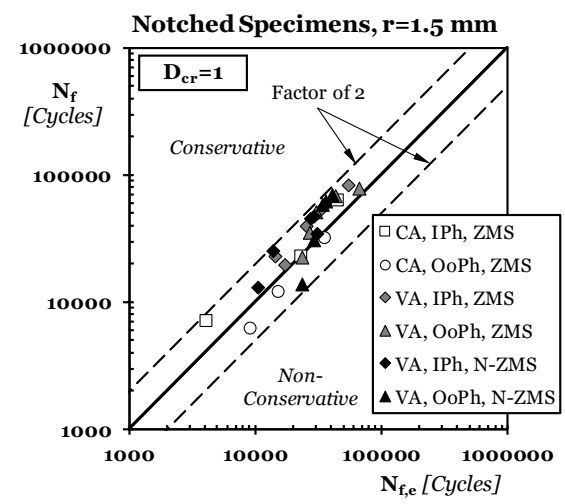

(c)

Notched Specimens, $r=6 \mathrm{~mm}$

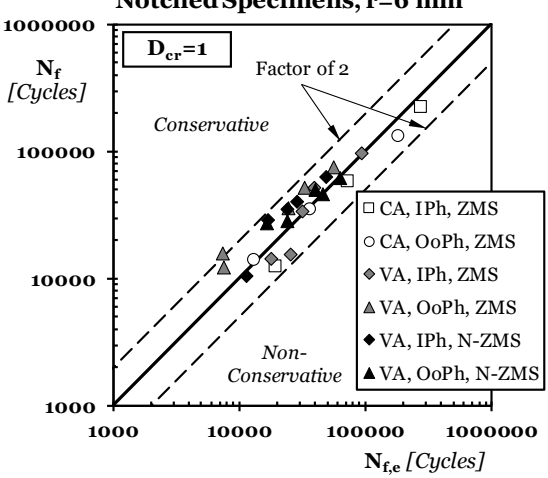

(e)
Plain Specimens

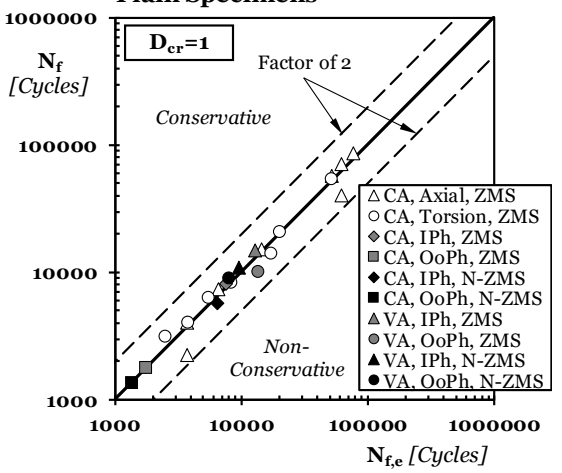

(b)

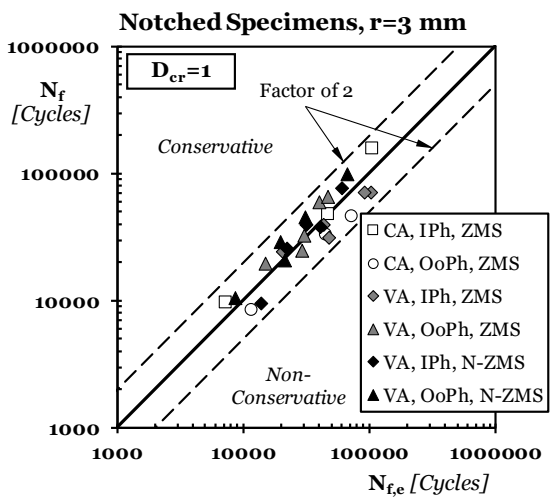

(d)

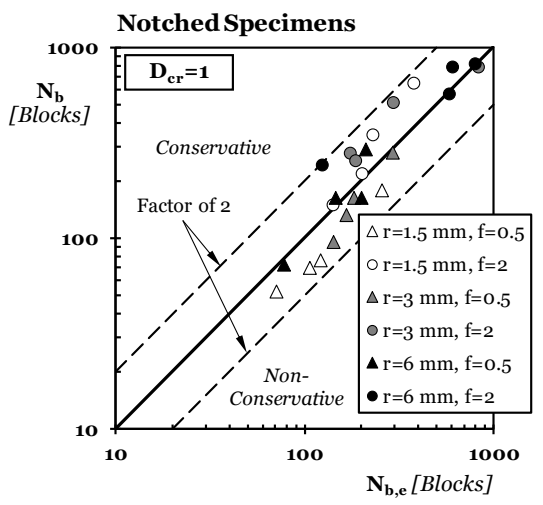

(f)

Fig. 6. Overall accuracy of the proposed multiaxial fatigue life estimation technique $(\mathrm{CA}=\mathrm{Constant}$ Amplitude; VA=Variable Amplitude; $\mathrm{IPh}=\mathrm{In}$-Phase; $\mathrm{OoPh}=90^{\circ}$ Out-of-Phase; $\mathrm{ZMS}=\mathrm{Zero}$ Mean Stress; N-ZMS=Non-Zero Mean Stress; $\mathrm{f}=$ ratio between the frequencies of the axial and torsional loading channels).

The accuracy of the design procedure schematically described in Figs 3 and 4 is summarised via the error charts of Figs $6 \mathrm{c}$ to $6 \mathrm{f}$, where, as per the plain specimens, the VA fatigue lifetime of the notched specimens was estimated by taking the critical value of the damage sum, $\mathrm{D}_{\mathrm{cr}}$, invariably equal to unity $[1,2]$. To conclude, it can be said that, according to these error chats, the use of our multiaxial fatigue life estimation technique was seen to 
result in a very high level of accuracy, with all the experimental points falling within an error factor of 2 .

\section{Conclusions}

The multiaxial fatigue assessment methodology proposed in the present paper was seen to be successful in estimating lifetime of notched metallic materials subjected to both CA and VA load histories. In particular, a remarkably high level of accuracy was systematically reached independently of sharpness of the notch being designed, degree of non-proportionality of the load history being post-processed, mean stress level, and ratio between the frequencies of the applied loading components.

Accordingly, it is possible to conclude by saying that this novel approach can be treated as a powerful tool suitable for performing the multiaxial fatigue assessment of real notched structural components, the key advantage being that it can be applied by directly postprocessing the relevant stress/strain fields determined from elasto-plastic numerical models solved using commercial FE codes.

\section{References}

1. R.I. Stephens, A. Fatemi, R.R. Stephens, H.O. Fuchs, Metal Fatigue in Engineering, $2^{\text {nd }}$ Edition, John Wiley \& Sons, USA (2001)

2. D.F. Socie, G.B. Marquis, Multiaxial Fatigue, SAE, Warrendale, PA, USA (2000)

3. L. Susmel, Multiaxial Notch Fatigue: from nominal to local stress-strain quantities. Woodhead \& CRC, Cambridge, UK (2009)

4. S.M. Tipton, J.W. Fash, Multiaxial fatigue life prediction for the SAE specimen using strain based approaches. In: Multiaxial fatigue: analysis and experiments, Edited by G. E. Leese and D. F. Socie, SAE AE-14, pp. 67-80 (1989)

5. L. Susmel, G. Meneghetti, B. Atzori, A novel Multiaxial Fatigue Criterion to predict Lifetime in the Low/Medium-Cycle Fatigue Regime. Part II: Notches. Trans ASME, J Eng Mater Technol, 131: 021010-1/8 (2009)

6. N. Gates, A. Fatemi, Notched fatigue behavior and stress analysis under multiaxial states of stress. Int J Fatigue, 67: 2-14 (2014)

7. L. Susmel, D. Taylor, An elasto-plastic reformulation of the Theory of Critical Distances to estimate lifetime of notched components failing in the low/medium-cycle fatigue regime. Trans ASME, J Eng Mater Technol, 132: 021002-1/8 (2010)

8. L. Susmel, D. Taylor, Estimating lifetime of notched components subjected to variable amplitude fatigue loading according to the elasto-plastic Theory of Critical Distances. Trans ASME, J Eng Mater Technol 137: 011008-1/15 (2015)

9. L. Susmel, G. Meneghetti, B. Atzori, A simple and efficient reformulation of the classical Manson-Coffin curve to predict lifetime under multiaxial fatigue loading. Part I: plain materials. Trans ASME, J Eng Mater Technol, 131: 021009-1/9 (2009)

10. L. Susmel, A simple and efficient numerical algorithm to determine the orientation of the critical plane in multiaxial fatigue problems. Int J Fatigue, 32: 1875-1883 (2010)

11. Y. Wang, L. Susmel, The Modified Manson-Coffin Curve Method to estimate fatigue lifetime under complex constant and variable amplitude multiaxial fatigue loading. Int $\mathrm{J}$ Fatigue, 83: 135-149 (2016) 